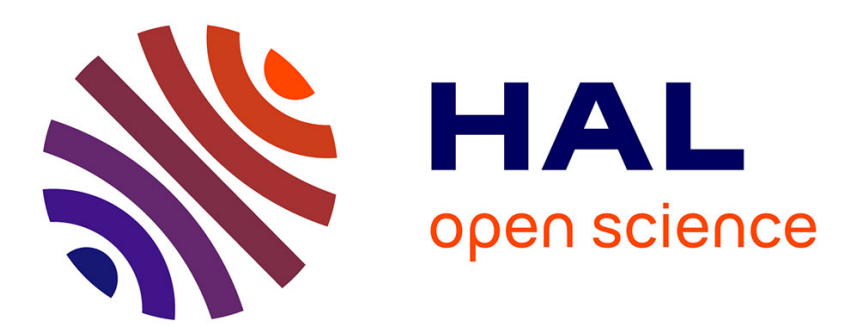

\title{
Le management au défi du stress des professionnels dans les établissements d'accueil des personnes âgées dépendantes
}

\author{
Alan Vinot, Didier Vinot
}

\section{- To cite this version:}

Alan Vinot, Didier Vinot. Le management au défi du stress des professionnels dans les établissements d'accueil des personnes âgées dépendantes. Journal de gestion et d'économie médicales, 2017, 35 (4), pp.159-177. 10.3917/jgem.174.0159 . hal-01876707

\section{HAL Id: hal-01876707 https://univ-lyon3.hal.science/hal-01876707}

Submitted on 18 Mar 2020

HAL is a multi-disciplinary open access archive for the deposit and dissemination of scientific research documents, whether they are published or not. The documents may come from teaching and research institutions in France or abroad, or from public or private research centers.
L'archive ouverte pluridisciplinaire HAL, est destinée au dépôt et à la diffusion de documents scientifiques de niveau recherche, publiés ou non, émanant des établissements d'enseignement et de recherche français ou étrangers, des laboratoires publics ou privés. 


\section{LE MANAGEMENT AU DÉFI DU STRESS DES PROFESSIONNELS DANS LES ÉTABLISSEMENTS D'ACCUEIL DES PERSONNES ÂGÉES DÉPENDANTES}

Alan Vinot, Didier Vinot

\section{ESKA | «Journal de gestion et d'économie médicales »}

2017/4 Vol. 35 | pages 159 à 177

ISSN 2262-5305

ISBN 9782747227513

\section{Article disponible en ligne à l'adresse :}

https://www.cairn.info/revue-journal-de-gestion-et-d-economiemedicales-2017-4-page-159.htm

\section{Pour citer cet article :}

Alan Vinot, Didier Vinot «e management au défi du stress des professionnels dans les établissements d'accueil des personnes âgées dépendantes », Journal de gestion et d'économie médicales 2017/4 (Vol. 35), p. 159-177.

DOI 10.3917/jgem.174.0159

Distribution électronique Cairn.info pour ESKA.

(C) ESKA. Tous droits réservés pour tous pays.

La reproduction ou représentation de cet article, notamment par photocopie, n'est autorisée que dans les limites des conditions générales d'utilisation du site ou, le cas échéant, des conditions générales de la licence souscrite par votre établissement. Toute autre reproduction ou représentation, en tout ou partie, sous quelque forme et de quelque manière que ce soit, est interdite sauf accord préalable et écrit de l'éditeur, en dehors des cas prévus par la législation en vigueur en France. Il est précisé que son stockage dans une base de données est également interdit. 


\title{
Le management au défi du stress des professionnels dans les établissements d'accueil des personnes âgées dépendantes
}

\section{The management in the challenge of the stress of the professionals in nursing home}

\author{
Alan VINOT ${ }^{1 *}$ et Didier VINOT ${ }^{2 *}$ \\ ${ }^{1}$ Docteur en Sciences de Gestion, Directeur Général de la Fondation Bompard, \\ École des Hautes Études en Santé Publique, EHESP (EA 7348 MOS), vinotalan@hotmail.com \\ ${ }^{2}$ Professeur des universités, co-directeur de la chaire Valeur du soin centré patient, IAE Lyon, MAGELLAN \\ EA3713, Université Jean Moulin Lyon 3, didier.vinot@univ-lyon3.fr \\ * Les auteurs n'ont pas de lien de famille connu.
}

\section{RÉSUMÉ}

Objectif : L'objectif de cet article est de proposer un outil de mesure de perception du stress au sein de structures médico-sociales et à en analyser les résultats.

Champs de l'étude : Cette recherche a été menée au sein de 26 Etablissements Hébergeant des Personnes Agées Dépendantes (EHPAD) répartis sur toute la France et intègre des structures citadines, rurales, de tailles, de statuts et de niveau de dépendance différents.

Population étudiée : Tous les professionnels présents au sein de l'établissement au moment de l'étude.

Méthode : Le questionnaire a été construit à partir de de trois structures pilote. Chaque échelle a été testée en fonction des alphas de Cronbach, $\alpha$. Les résultats ont été exploités via une régression linéaire multiple de la fonction perception du stress.

Résultats : La recherche porte sur 914 professionnels qui ont participé à cette étude. Le questionnaire comporte 42 questions portant sur des variables spécifiques aux conditions de travail et d'autres plus spécifiques aux critères identifiés lors des phases de pré test. L'alpha de Cronbach fait apparaître un coefficient de .893, quant aux 13 questions relatives aux conditions de travail, et de .848 quant aux 21 questions spécifiques au stress identifiées en focus group. Les résultats mettent en exergue 8 facteurs propres au rythme de travail, à la clarté des missions, 
aux interruptions des activités, au sens au travail, à la relation avec les résidents ou avec les collègues, aux ordres contradictoires et à l'encadrement.

Conclusions : Certains facteurs tels que la relation avec les familles n'interviennent pas dans l'analyse des causes du stress, mettant en avant des facteurs organisationnels plus marqués, qui sont de réels leviers d'actions pour le manager.

Mots-clés : stress, performance, management, organisation, EHPAD.

\section{ABSTRACT}

The main objective of this article is to propose a management tool for perception of the job stress within medical and social structures and to analyze the results.

This research was led within 26 elderly-care facilities for dependent persons throughout France.

Method: The questionnaire was built from three experimental structures. Every scale was tested according to the alphas of Cronbach. The results were exploited via a multiple linear regression of the functions of different perceptions of the stress job.

The research concerns 914 professionals who participated in this study. The questionnaire contains 42 questions concerning specific variables in the conditions of work and others more specific to the criteria identified during the phases of meadow make out a test. The alpha of Cronbach reveals a coefficient of .893, as for 13 relative questions in the conditions of work, and of .848 as for 21 questions specific to the stress identified in focus group.

The results highlight 8 factors in connexion with the rhythm of the work, the clarity of the missions, the interruptions of the activities, the direction in the work, the contradictory orders and the general management.

Conclusions: Some factors such as the relation with families do not intervene in the analysis of the causes of the stress, putting in front of the more marked organizational factors, which are real levers of actions for the management.

Keywords: job stress, performance, management, organization, elderly-care facilities. 


\section{INTRODUCTION}

Le lien entre le bien-être d'un résident accueilli dans un établissement d'accueil pour personnes âgées dépendantes, de sa famille et celui du professionnel qui exerce dans cette même institution est souvent présupposé comme évident. Mais qu'en est-il vraiment ? Dans quelle mesure le bien-être au travail est-il lié à la satisfaction des résidents et à une faible perception du stress par ses professionnels?

Chacun est tenté de répondre spontanément selon sa propre représentation ou son expérience à ces questions mais force est de constater l'impossibilité d'une réponse consensuelle, tant la subjectivité des notions de performance et plus particulièrement de stress sont considérables. L'enjeu de cette recherche est donc d'une part de discerner les facteurs qui influencent la perception du stress et d'autre part de discuter de nouveaux leviers managériaux adaptés au contexte sanitaire et médico-social et plus particulièrement celui des Etablissements Hébergeant des Personnes Agées Dépendantes (EHPAD). Cette question est cruciale au sein d'un secteur en tension. Tension dans la souffrance aussi bien physique que psychique exprimée par ses professionnels et tension propre aux difficultés exprimées par les résidents, leurs familles et leurs proches.

Cette recherche réalisée en partenariat avec l'Agence Nationale d'Appui à la Performance (ANAP), s'est donnée pour but de mesurer la perception du stress par les professionnels et d'en analyser les facteurs au sein des 26 EHPAD. A partir de la littérature existante, un questionnaire spécifique au secteur sanitaire et médio social visant à évaluer la perception du stress a été élaboré. Après une validation de l'outil proposé dans trois établissements pilotes, cet outil a été déployé en partenariat avec l'ANAP au sein d'un panel d'établissements des secteurs privés à but non lucratif, et des fonctions publiques hospitalières et territoriales, répartis sur l'ensemble du territoire français. Une régression linéaire multiple de la fonction perception du stress a permis de mettre en exergue des facteurs d'origine professionnelle pouvant constituer un levier managérial. Dans cette optique, après avoir explicité la notion de perception du stress, ses outils d'évaluation et ses postulats de facteurs explicatifs, nous présenterons la méthodologie retenue, et analyserons les résultats obtenus de cette étude qui s'est déroulée sur trois années, de 2011 à 2013.

\section{CADRE CONCEPTUEL ET MÉTHODOLOGIE}

\subsection{Approche théorique du stress en situation de travail}

Utilisée pour la première fois par Robert Hoocke, biologiste et physicien du XVII ${ }^{e}$ Siècle dans une loi qui décrivait la relation linéaire entre l'élongation d'un ressort (Strain) et la force à laquelle il était soumis (Stress), la notion de stress a été importée par H. Selye au début du $X^{\mathrm{e}}$ Siècle dans le champ de la physiologie et de la médecine (Selye, 1936). Elle a connu depuis cette date un succès considérable (Timsit-Berthier, 2014). Par exemple, entre 1970 et 2007, le «stress » a été l'objet de 210000 articles scientifiques publiés aussi bien dans des revues de médecine et de biologie que dans des Journaux de psychologie, de sociologie, d'économie, de droit et de politique (Le Modal, 2007, cité par Tilsit-Berthier, 2014), jusqu'à se généraliser dans le langage du quotidien où le nom «stress » est devenu un adjectif puis un verbe.

Selon Selye, les réactions dues au stress sont non spécifiques, globales et quasi automatiques. 
Cette idée de «non spécificité » a pu marquer une rupture avec les conceptions pasteuriennes qui prédominaient l'époque et qui posaient l'existence d'une relation linéaire entre un agent pathogène particulier et les réactions spécifiques de l'organisme. Par ailleurs, ces réactions de stress visent à supprimer les déséquilibres biologiques provoqués par l'agression et à ramener les variables biologiques à leurs valeurs initiales. C'est pourquoi Selye les a qualifiées de «syndrome général d'adaptation », en notant que ces réactions peuvent être divisées en trois phases successives, en fonction de leur durée : une phase d'alarme brève, pendant laquelle existe une mobilisation intense d'énergie, une phase de résistance qui peut durer plusieurs semaines, puis, éventuellement une phase d'épuisement, qui menace la survie de l'organisme.

Quittant le domaine purement médical, Lazarus et Folkman (1984) ont mis en avant la notion d'adaptation, qui est à distinguer de celle de coping, dans la mesure où elle inclut tous les modes de réactions des organismes vivants face aux changements environnementaux alors que le coping est spécifique à l'homme et ne concerne que des réponses aux variations de l'environnement perçues comme stressantes. Comme le démontrent Kolek, Bruchon-Schweitzer et Bourgeois (2003), il existe deux types de stratégies de coping : l'une centrée sur le problème, et l'autre centrée sur les réponses émotionnelles induites par la situation.

Dans le monde du travail, l'impact du milieu professionnel a très tôt été mis en évidence comme facteur explicatif du stress des personnes. Dans ce cadre, Karasek (1979) a défini les déterminants de stress au travail à travers l'interaction de deux variables : la latitude de décision et les demandes environnementales. Karasek et Theorell (1990) ont complété cette interaction par l'apport d'un nouveau déterminant qu'est le soutien social des collègues et des supérieurs correspondant à la mesure de l'intensité perçue de stress. Ils considèrent ainsi qu'une forte demande psychologique, une faible latitude décisionnelle, et peu de soutien social au travail constituent la configuration «optimale » générant du stress. Cette approche, souligne l'écart entre la situation et l'individu, permettant ainsi de combler le manque de l'approche transactionnelle en tenant compte de l'organisation à travers la latitude décisionnelle et le soutien social au travail.

A la suite de ces travaux, Hellemans et Karnas (1999) ont réalisé une analyse factorielle des trois dimensions afin de les décliner en 6 facteurs que sont : la latitude de décision et l'apprentissage, la pression du temps et le dérangement, le soutien social du supérieur et le soutien social des collègues, dans le but d'affiner les profils en fonction du degré du stress perçu. Ils concluent que plus les personnes perçoivent un stress élevé, moins elles disposeraient de ressources individuelles et sociales. Détaillant l'intensité du stress produit, le modèle proposé par Siegrist (1996) détermine le niveau de stress en fonction d'un déséquilibre entre la mesure des efforts fournis par l'individu au travail et les rétributions correspondantes. Ce déséquilibre entraînerait un «sur-investissement » de la part de l'individu pouvant engendrer du stress.

Dejours (2000) reprend cette notion en parlant de contribution-rétribution comme facteur de mobilisation. Deux types d'efforts se distinguent alors dans ce modèle : les efforts « extrinsèques », assez similaires au facteur de demande de Karasek et les " efforts intrinsèques » de surinvestissements, plutôt relatifs à un besoin d'approbation, d'hostilité, de compétitivité. C'est pourquoi une surexposition à ces types d'efforts représente un risque potentiel de stress. Tout comme le modèle de Karasek, celui de Siegrist est souvent employé dans les 
études épidémiologiques sur le stress au travail. Ses limites ont notamment, été précisées par Legault et Belarbi (2006) qui questionnent le lien entre l'autonomie opérationnelle et les conditions favorables à la santé psychologique. Ainsi, l'autonomie accordée aux professionnels en échange de l'engagement à atteindre des objectifs dont ils se rendent responsables est génératrice d'arbitrages internes (Gadrey, 2000). Par le chevauchement des projets dans les structures, notamment médico-sociales, les professionnels se voient imposer de considérables demandes concurrentes qu'ils doivent mesurer. Ils ont alors l'obligation d'établir eux-mêmes l'ordre de priorités en cas de conflits de tâches, au risque de s'exposer à la critique des décisions prises. Il importe donc de distinguer l'autonomie décisionnelle qui favorise l'épanouissement, de celle qui mène à l'ambiguïté de rôles.

Gintrac (2011), dans son état des lieux sur le stress dit organisationnel, approfondit les approches de Karasek, Theorell (1990) et Siegriest (1996). Selon lui, les individus ne sont pas égaux devant les facteurs de stress dans la mesure où dans une même situation, les réactions peuvent diverger. Il établit également que les tensions extérieures influent sur l'efficacité au travail précisant qu'un stress au travail peut, au moins en partie, ne pas avoir sa source dans des facteurs liés au travail. Ainsi, la distinction entre causes professionnelles du stress et causes privées serait pratiquement impossible à réaliser.

Plusieurs études complémentaires tendent par ailleurs à le démontrer : Morin et Gagne (2009), Dupuis et al. (2009), Moisson et al., (2010). Plus spécifiquement, Carrier et al., (2012), ont mis en évidence une relation positive entre le conflit entre vie professionnelle et vie personnelle et l'épuisement d'origine professionnelle.
De manière plus générale et sur cette question des facteurs propres à la sphère personnelle, la littérature démontre que le conflit travail-famille joue un rôle médiateur entre les facteurs de stress, le stress lui-même, et par extension la santé au travail (Cooper et al., 1992 ; Demerouti et al., 2004). L'effet médiateur du conflit travail-famille entre les exigences de l'emploi et du stress d'origine professionnelle a notamment pu être démontré par Voydanoff (2005). Le conflit travail-famille influence la relation entre les caractéristiques du travail et notamment la charge de travail dite quantitative et la santé psychologique (Geurts et al., 2003).

\subsection{Outil d'évaluation des facteurs de stress professionnel et construction du questionnaire}

L'approche transactionnelle de Lazarus et Folkman appréhende les mécanismes psycho-cognitifs du stress, et distingue l'évaluation primaire, et l'évaluation secondaire. Plusieurs échelles ont été élaborées, mais au regard des apports de Guillet (2012), nous avons retenu l'échelle PSS10, échelle unidimensionnelle qui possède d'excellentes qualités psychométriques. Afin de mesurer l'évaluation secondaire, l'échelle PSS10 de Cohen et al. (1983) nous a semblé pertinente pour mesurer la fréquence avec laquelle les situations de vie - ici de travail - sont perçues comme menaçantes, ou stressantes pour le professionnel en EHPAD. De nombreux travaux ont été engagés concernant la structure factorielle de cette échelle, et la grande majorité retient deux facteurs latents. Le premier facteur a été interprété en termes de perception de débordement. Il s'agit ici d'une réponse émotionnelle et/ou cognitive face à des sollicitations ponctuelles ou chroniques. Le second, développé par Bellinghausen et al. (2009), a été interprété en termes d'efficacité 
personnelle perçue, c'est-à-dire la croyance qu'a un individu d'avoir les capacités de surmonter les difficultés pour atteindre son objectif.

Afin de mesurer la perception du stress des professionnels, nous avons procédé à une combinaison de deux mesures, l'une subjective à travers une auto évaluation du stress de 1 à 10 et l'autre plus formelle via l'outil PSS10, «Global measure of perceived stress ». La différence entre ce que les personnes pensent ressentir comme un stress, et le stress qu'elles vont réellement percevoir est donc mesurée. C'est pourquoi nous avons choisi de mesurer cette notion complexe de deux façons et d'observer ainsi les différences pouvant exister entre leur perception subjective et la mesure du stress perçu par les salariés selon une échelle de mesure scientifiquement.

Enfin, la question de la méthodologie de recueil et notamment ses biais étant prépondérante, les travaux de Steiler et Rosnet (2011) sur la mesure du stress professionnel revêtent un intérêt certain. La méthodologie, via cette interrogation sur la question de la perspective - y compris sur la considération même de la notion de stress au travail, tel que proposé par Ponnelle et Lancry (2002) - fut au cœur de notre réflexion. A partir de ces travaux, nous avons élaboré un questionnaire ad hoc qui intègre les spécificités des structures sanitaires et médicosociales en prenant en compte, notamment, la dépendance physique, la dépendance psychique mais aussi la fin de vie. Celui-ci a été testé à travers 3 groupes au sein de trois EHPAD de taille différente, ruraux, citadins et de niveau de dépendance très aléatoire. Chaque groupe représentait $40 \%$ des effectifs, la proportion de chaque catégorie socio-professionnelle étant elle-même conservée. Huit rencontres ont été conduites avec chaque groupe afin de cerner les facteurs de stress professionnel dans l'exercice de l'activité du professionnel et de l'équipe pluridisciplinaire. Ces entretiens ont été complétés par deux rencontres avec les instances représentatives du personnel, la médecine du travail, l'agence régionale d'amélioration des conditions de travail ainsi que trois administrateurs du conseil d'administration. Fort de ces contributions, un questionnaire a été proposé et testé. Les résultats étant concluants en termes de robustesse et de fiabilité, celui-ci a été étendu au niveau national via le partenariat avec l'Agence Nationale d'Appui à la Performance. 10 items globaux ont été retenus : la communication, l'organisation, le management, la relation avec les résidents, la relation avec les familles, la pénibilité au travail, la charge de travail, l'ergonomie, la cohésion d'équipe et la dimension individuelle.

\subsection{Définition des hypothèses de recherche}

La phase exploratoire nous a conduits à définir 23 hypothèses dont 8 portent plus particulièrement sur la notion de rôle.

Les travaux de Biddle (1979) ont montré que la notion de rôle s'articule autour de deux éléments clefs : les attentes réciproques relatives aux activités et les comportements relationnels exercés par un individu dans un contexte organisationnel donné. Le concept de rôle organisationnel est donc essentiellement lié aux apports de Kahn et al. (1964) puis Katz et Kahn (1966) qui distinguent dans leur modèle, les attentes de rôle, le rôle transmis, le rôle perçu et le rôle joué. Ainsi, les inter relations entre les rôles créent des transmissions incohérentes qu'ils qualifient de conflits de rôle. Ils évoquent ainsi le conflit « inter émetteurs », lorsque deux personnes émettent des attentes contradictoires vis-à-vis d'une seule et même personne. Ce sont les hypothèses que nous avons identifiées et qui portent sur les ordres contradictoires 
issus de deux canaux hiérarchiques différents ( $\mathrm{H} 3 b)$, la communication $(\mathrm{H} 3 \mathrm{c})$ et les relations (H3d) avec les collègues. Lorsque ces attentes contradictoires sont formulées par un responsable hiérarchique unique, alors ils évoquent un « conflit intra-émetteur». Dans notre recherche, cela correspond à l'hypothèse $(\mathrm{H} 3 \mathrm{a})$ qui porte directement sur la communication avec le responsable direct.

Katz et Kahn (1966) abordent par ailleurs deux autres conflits de rôles possibles qui ne portent plus sur le rôle joué mais sur le conflit « individu-rôle » qui peut apparaître lorsque le professionnel doit jouer en même temps deux rôles incompatibles entre eux. Nous avons alors défini l'hypothèse relative à la clarté de la mission $(\mathrm{H} 3 \mathrm{e})$. Ils distinguent également l'exemple de l'exercice d'un rôle qui serait contraire à ses propres attentes. Cela se traduit dans notre recherche dans les hypothèses relatives à l'ambiguiité de la confrontation à la vie et à la mort (H3f). De la même manière, la non adhésion aux valeurs de l'établissement (H3g) ou l'absence de sens perçu à son travail (H3h) peuvent aussi être l'objet d'un rôle contraire à ses propres attentes. Enfin, et même si cette forme est contestée Murphy et Gable (1988) puis Schaubroek Cotton, Jennings (1989), distinguent la «surcharge de rôle » comme une quatrième forme possible de conflit de rôles. Nous n'avons pas, au regard des critiques formulées mais aussi des entretiens menés, émis d'hypothèse quant à cette forme de conflit de rôle.

Ces apports précisent également notre ancrage dans une approche multidimensionnelle qui considère que le professionnel ne se situe pas dans un rôle, mais dans un ensemble de rôles dont les conflits pourront au-delà des comportements attendus porter sur les interactions des multiples rôles. Alis (1997) a démontré que les conflits de rôle ne sont pas uniquement des conflits objectifs et dysfonctionnels, mais bien des conflits subjectifs vécus par l'individu. Il a mis en exergue que les professionnels ne sont pas prisonniers des rôles qui leurs sont prescrits mais disposent d'une marge d'adaptation et mettent en place des processus de régulation autonome. A la suite de Perrot (2005), nous avons privilégié une approche multidimensionnelle du concept de conflit de rôle, qui ne repose pas uniquement sur des conflits objectifs et dysfonctionnels, mais également sur des conflits subjectifs vécus par l'individu. Le conflit de rôle, comme l'avance Loubès (1997), s'avère donc être la perception de la présence de deux ou plusieurs ensembles de pressions et/ou d'attentes relatives au travail, telle que l'adaptation de l'une entrave celle des autres. Par ailleurs, la mesure de la clarté dans la mission peut être rapprochée de la notion d'ambiguïté de rôle, plus nuancée que celle de conflit de rôle.

Parallèlement à la notion de rôle, quinze autres hypothèses ont été définies via les focus groups menés au sein des trois structures pilotes. Une attention particulière, du fait des retours notamment des soignants, a été portée sur la relation Professionnel - Résident en identifiant l'incidence du comportement du résident comme vecteur de perception de stress pour le professionnel (H1a) et le comportement de la famille (H1b). Par comportement, nous avons identifié aussi bien les attitudes comportementales que les propos, qu'ils soient oraux ou écrits. Dans cette continuité et face au développement de la démarche qualité au sein des structures médico-sociales, nous avons identifié deux hypothèses relatives à l'expression de la satisfaction ou de l'insatisfaction du résident $(\mathrm{H} 1 \mathrm{c})$ et de la famille (H1d). Enfin, la question de la présence de la famille, notamment lors des soins, comme vecteur de perception de stress a été exprimée assez fortement par les professionnels, d'où la définition de l'hypothèse H1e. 
La dimension organisationnelle a été, lors des entretiens avec les professionnels, une dimension souvent associée à une source de perception du stress. Plusieurs points ont alors été mis en exergue afin de tester sept hypothèses et notamment la perception de l'organisation par le salarié (H2a), le rythme de travail (H2b) exprimé par toutes les catégories socio professionnelles, la latitude de décision (H2c) au sens de Karasek et Theorell (1990), la remontée d'information à la hiérarchie (H2d), les interruptions fréquentes et sans lien direct avec la mission assignée au professionnel (H2e), le ressenti d'iniquités ( $\mathrm{H} 2 \mathrm{f})$, l'ambiance de travail et notamment la question de la tension au sein de l'équipe (H2g).

Les questions matérielles ont aussi été exprimées et notamment l'adaptation du matériel à une dépendance grandissante au sein des établissements, d'où la définition de l'hypothèse ( $\mathrm{H} 4 \mathrm{a})$. Par ailleurs, la question du manque de confort des espaces de repos a été exprimée. Cela trouve son explication au regard, de l'accroissement des horaires de travail dits « en coupé » et qui induisent pour celles et ceux éloignés de leur domicile à devoir rester sur leur lieu de travail pendant cette période d'inactivité. De ce fait, l'hypothèse (H4b) a été proposée.

Enfin, et même si nous avions au préalable indiqué que cette étude sur les facteurs vecteurs de perception du stress portait sur la dimension professionnelle, nous avons au regard des retours en focus group identifié plusieurs facteurs relatifs à la sphère individuelle que sont la tranche d'âge, le sexe, la fonction et l'ancienneté, via H5.

La figure suivante synthétise les hypothèses de recherche formulées :

Figure 1 : Hypothèses de recherche

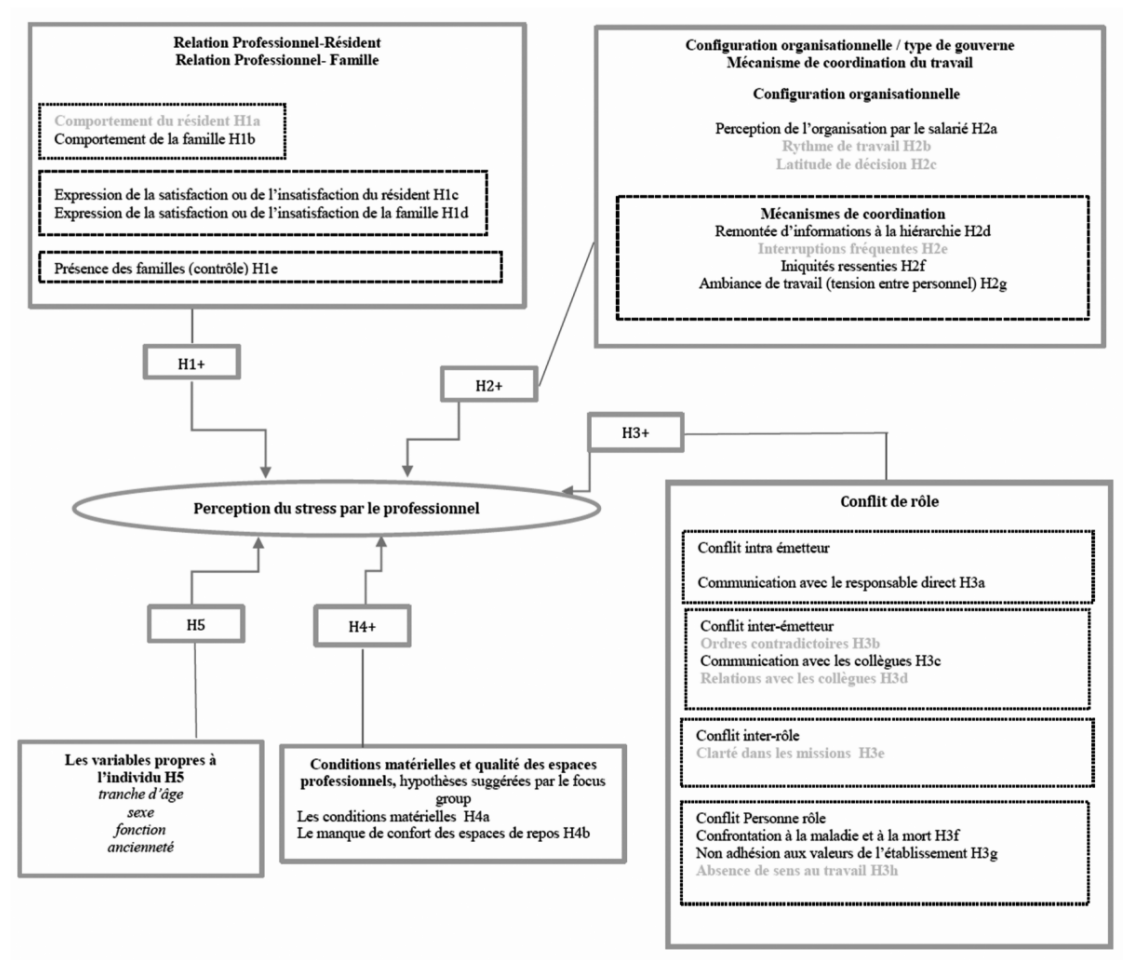


Tableau 1 : Caractéristiques des structures de la base de données

\begin{tabular}{|l|c|c|c|c|c|}
\hline \multicolumn{1}{|c|}{ Département } & Statut & Nb de salariés & Département & Statut & Nb de salariés \\
\hline Ile et Vilaine & FPH & 93 & Maine et Loire & PNL & 48 \\
\hline Var & FPT & 58 & Ariège & PNL & 25 \\
\hline Maine et Loire & FPT & 48 & Manche & PNL & 48 \\
\hline Ile et Vilaine & FPT & 29 & Gard & PNL & 41 \\
\hline Ile et Vilaine & FPT & 38 & Hérault & PNL & 45 \\
\hline Ile et Vilaine & FPT & 55 & Gard & PNL & 33 \\
\hline Ile et Vilaine & FPT & 65 & Hérault & PNL & 49 \\
\hline Var & PNL & 60 & Aude & PNL & 44 \\
\hline Vaucluse & PNL & 41 & Moselle & FPH & 36 \\
\hline Rhône & PNL & 44 & Ile et Vilaine & FPT & 34 \\
\hline Moselle & PNL & 45 & Côtes d'Armor & PNL & 58 \\
\hline Moselle & PNL & 48 & Bas Rhin & FPH & 35 \\
\hline Moselle & PNL & 50 & Bas Rhin & FPH & 33 \\
\hline
\end{tabular}

FPH : Fonction Publique Hospitalière, FPT : Fonction Publique Territoriale, PNL : Privé Non Lucratif

\subsection{Population et test de fiabilité}

Un questionnaire a été administré auprès de 914 professionnels répartis sur 26 établissements du territoire français. L'intégration d'établissements de taille différente, de niveaux de dépendance différents aussi bien physique que psychique, citadins, ruraux, également répartis sur le territoire a été effectuée. Le tableau ci-dessous en explicite les caractéristiques principales.

Cette population, répartie sur des territoires variés est représentée, de la façon suivante :

Figure 2 : Typologie de la population étudiée
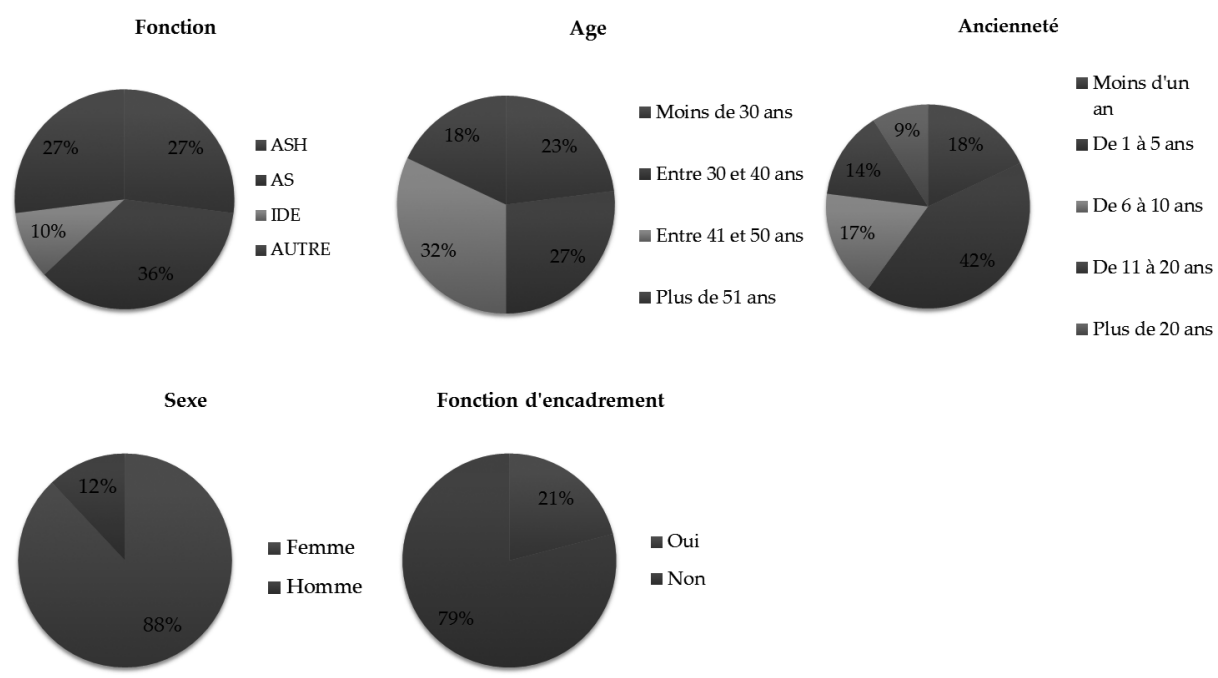
Tableau 2 : Mesure de l'alpha de Cronbach

\begin{tabular}{|l|c|c|}
\hline \multicolumn{1}{|c|}{ Variables « Conditions de travail » } & Alpha de Cronbach & Nombre d'éléments \\
\hline Questions 9 à 21 &, 893 & 13 \\
\hline Variables spécifiques au stress & Alpha de Cronbach & Nombre d'éléments \\
\hline Questions 22 à 42 &, 848 & 21 \\
\hline
\end{tabular}

914 questionnaires ont été retournés. L'alpha de Cronbach fait apparaître un coefficient de .893 , quant aux 13 questions relatives aux conditions de travail, et de .848 quant aux 21 questions spécifiques au stress.

L'homogénéité de l'instrument et par extension sa consistance interne est donc vérifiée, ce qui a permis de procéder avec confiance à l'analyse des résultats.

\section{RÉSULTATS : LES FACTEURS PROFESSIONNELS D'INFLUENCE DE LA PERCEPTION DU STRESS}

\subsection{Régression linéaire multiple de la fonction perception du stress}

Afin de déterminer l'influence des facteurs sur le niveau de stress des salariés, nous avons procédé à une régression linéaire permettant ainsi de définir la part du stress qui peut être expliquée par les facteurs professionnels alors identifiés dans le cadre de l'étude. Les résultats de cette régression font ressortir des facteurs qui semblaient pourtant ne pas être mis en cause lors des entretiens menés en focus group. A l'inverse, elle ne fait pas, par exemple, ressortir des facteurs directement liés à une relation vécue difficilement par le professionnel avec les familles. Nous avons utilisé la méthode « Pas à pas », dans laquelle les variables indépendantes (variables de stress) ont été introduites une par une dans la régression et ont été éliminées si elles ne contribuaient plus significativement à la régression.

Notre volonté a donc été d'intégrer toutes les variables afin de ne négliger aucun aspect, dans la mesure où certaines caractéristiques peuvent grandement influencer le niveau de stress au regard du contexte de la structure.

La régression a permis de ressortir 8 variables, avec un $R 2$ ajusté de .270 , une variation de Fisher de 4,885 et avec un seuil de significativité inférieur à $5 \%$.

Ce modèle a mis en exergue les facteurs « Charge de travail », " Organisation du travail », « Pénibilité », « Sens au travail », «Management», « Relation avec les résidents », « Cohésion » et « encadrement ».

Ainsi la fonction perception du stress peut être proposée par la droite de régression suivante :

Fonction Stress $(Y)=6,412$ (Constante) $+.698 \times$ « Rythme de travail +.436 $x$ «Clarté des missions» $+.612 x$ «Interruptions » +.679 $x$ « Sens au travail » $+.538 \times$ 《 Relation avec les résidents » $+.475 \mathrm{x}$ 《 Relation avec les collègues" $+.433 x$ «Ordres contradictoires » -
$.827 x$ « Encadrement $»+.901$ (Risque d'erreur) $+\beta$ (autres facteurs de stress non contrôlés) 
Tableau 3 : Mesure des coefficients de corrélation et de détermination

\begin{tabular}{|ccrrrrr|}
\hline Modèle & $R$ & & R-deux & R-deux ajusté & Variation de & Durbin et \\
& & & & & & \\
& & & & & & \\
1 &, 380 &, 144 &, 144 & $154,042^{* * *}$ & & \\
2 &, 445 &, 198 &, 196 & $60,563^{* * *}$ & & \\
3 &, 474 &, 224 &, 222 & $31,041^{* * *}$ & & \\
4 &, 495 &, 245 &, 242 & $25,176^{* * *}$ & & \\
5 &, 508 &, 258 &, 254 & $15,908^{* * *}$ & & \\
6 &, 516 &, 267 &, 262 & $10,407^{* * *}$ & & \\
7 &, 522 &, 272 &, 267 & $7,226^{* * *}$ & & \\
8 &, 526 &, 276 &, 270 & $4,885^{* *}$ & 1,983 & \\
\hline
\end{tabular}

Tableau 4 : Facteurs de stress émergents du modèle

\begin{tabular}{|c|c|c|c|}
\hline \multirow[b]{2}{*}{ Modèle 8} & \multicolumn{3}{|c|}{ Erreur } \\
\hline & $\mathbf{A}$ & standard & $\mathbf{t}$ \\
\hline (Constante) & 6,412 & ,901 & $7,118 * * *$ \\
\hline CHARGE DE TRAVAIL « Mon rythme de travail me stresse » & 698 & 144 & $4,834 * * *$ \\
\hline $\begin{array}{l}\text { ORGANISATION «Le manque de clarté dans les missions de chacun a } \\
\text { tendance à me stresser » }\end{array}$ & ,436 &, 159 & $2,739 * * *$ \\
\hline $\begin{array}{l}\text { PÉNIBILITÉ « Les interruptions fréquentes durant mon temps de travail } \\
\text { ont tendance à me stresser » }\end{array}$ & ,612 & ,158 & $3,877 * * *$ \\
\hline $\begin{array}{l}\text { SENS AU TRAVAIL «Je n'arrive pas (ou plus) à trouver de sens à mon } \\
\text { travail et cela m'affecte » }\end{array}$ & ,679 &, 162 & $4,195 * * *$ \\
\hline $\begin{array}{l}\text { RELATION AVEC LES RÉSIDENTS «Certains comportements des } \\
\text { résidents ont tendance à me stresser (agressions physiques, verbales)» }\end{array}$ &, 538 &, 151 & $3,576 * * *$ \\
\hline $\begin{array}{l}\text { COHÉSION « Les relations avec mes collègues ont tendance à me } \\
\text { stresser» }\end{array}$ & ,475 &, 157 & $3,030 * * *$ \\
\hline $\begin{array}{l}\text { MANAGEMENT «Il arrive que je reçoive des ordres contradictoires et } \\
\text { cela me stresse » }\end{array}$ & ,433 &, 153 & $2,835 * * *$ \\
\hline ENCADREMENT «Avez-vous une fonction d'encadrement ?» &,- 827 & ,374 & $-2,210 * *$ \\
\hline
\end{tabular}

Ainsi, 27\% de la variation du stress professionnel des salariés peut être expliquée par les facteurs d'ordre professionnel identifiés, et notamment par les huit cités ci-dessus. De plus, nous remarquons que le fait d'encadrer d'autres professionnels (qu'on ait le statut cadre ou non) a tendance à diminuer le stress, (-827). 


\subsection{Le rythme de travail comme variable majeure de perception du stress}

Nous avions observé que la variable « Rythme de travail » était corrélée avec la variable dépendante «Autoévaluation du stress » $(-, 606)$, ce qui vient confirmer nos observations précédentes et renforcer le lien entre le rythme de travail et la perception du stress. Les travaux de Pronost et al. (2008) au sein de services en onco-hématologie aboutissent partiellement à ce même résultat dans le sens où les caractéristiques liées à l'environnement du travail sont significatives, en particulier le besoin de temps auquel ils ajoutent le besoin de reconnaissance. Ce facteur du rythme est lié au facteur « temps prescrit pour les tâches ».

La première explication tient à l'évolution des politiques et à la mission même des EHPAD. Les EHPAD ont une population dépendante qui ne cesse de croître avec des moyens relativement constants. Cette dépendance grandissante s'explique notamment par les politiques de maintien à domicile, par les progrès de la médecine mais force est de constater que la charge de travail dans les structures s'accentue et impacte bien logiquement le rythme imposé à chacun.

Pour de nombreux professionnels, ce rythme est donc source de stress perçu qui découle sur d'autres facteurs qui eux aussi ressortent dans la régression tels que les interruptions, le sens ou les relations avec les collègues. Nous rejoignons les travaux d'Aubry (2012), qui a expérimenté l'intégration des normes collectives du rythme de travail auprès d'aides-soignantes et pour qui l'intensification du travail dans les structures gériatriques conduit à une fragilisation des collectifs (d'où le lien avec le facteur « relation avec les collègues » de notre étude), voire à une individualisation du rapport aux enjeux organisationnels. Toujours selon Aubry, une telle individualisation peut conduire les nouvelles recrues à vivre une souffrance éthique (lien avec le facteur « sens » dans cette recherche) dû à la nécessité de réaliser des actes moralement condamnables (lien avec le facteur « ordres contradictoires ») dans l'appréhension de respecter les délais impartis.

\subsection{La clarté de la mission, l'adaptation à son activité et la recherche du sens comme variables de gestion de la perception du stress}

Quant à la question de l'organisation et plus particulièrement du facteur « clarté dans les missions de chacun ", il est intéressant de constater que ce n'est pas la répartition des rôles et tâches de chacun, la question de l'autonomie ou encore l'organisation même du travail qui ressortent de la régression, mais le manque de clarté dans les missions de chacun. Ces résultats laissent penser que le professionnel a conscience d'une organisation de son travail, de son rôle et des tâches qui lui sont impartis tout en percevant une relative autonomie. Le manque de clarté constitue donc le vecteur d'une perception du stress. Il se fait ressentir à deux niveaux : l'un relatif au delta demeurant entre le prescrit et le réel, et l'autre propre à un fonctionnement plutôt réactif que proactif des structures, demandant aux professionnels une constante capacité d'adaptation. D'où ce sentiment d'être confronté à une organisation dont les missions évoluent constamment. Nous ne sommes pas dans une approche mécanique mais systémique car il s'agit de la clarté de la mission sous-entendu, la dualité qu'il existe entre le réel et le prescrit en référence aux travaux de Dejours (2000). D'autant plus que l'approche individuelle de la priorité entre tâche et activité demeure éminemment subjective et fonction des individualités telles que le démontrent les théories relatives à la cohabitation d'approches générationnelles différentes (Pauget, 2012). 
Le test du facteur « interruptions au cours du temps de travail » a montré qu'il influe positivement sur la perception du stress. Ainsi, la fréquence des interruptions illustre le décalage entre les tâches et l'activité : plus elles sont fréquentes, plus elles témoignent d'une difficulté organisationnelle à s'adapter à son activité. La variable « adhésion aux valeurs de l'établissement », n'intervient pas dans la régression linéaire alors que celle « Je n'arrive pas (ou plus) à trouver un sens à mon travail et cela m'affecte » a une influence non négligeable. Dans nos résultats, la question du sens est donc plus importante en termes d'influence sur le stress perçu que le facteur lié à l'adhésion aux valeurs. D'où le lien avec les travaux de Codo (2012) qui a traité l'influence de l'engagement au travail sur le stress des managers intermédiaires dans des collectivités territoriales en démontrant que le dévouement et l'absorption au travail affectent le stress. Par ailleurs, ce résultat peut également s'expliquer par la situation difficile que vivent les professionnels du secteur médicosocial, difficulté qui s'exprime tant dans la stabilité de l'emploi que dans le salaire et dans les conditions de travail. La question de la valeur n'est donc pas un réel facteur propre à influencer la perception du stress du professionnel contrairement au sens au travail.

\subsection{Des relations du professionnel avec les familles et avec le groupe qui n'expliquent pas le stress du professionnel}

Le critère « comportement des familles », à l'inverse du critère " comportement des résidents » ne ressort pas dans la régression. Cela peut s'expliquer d'une part par une confrontation beaucoup plus occasionnelle aux familles qu'aux résidents et d'autre part par la capacité du professionnel à mettre une distance avec les familles en cas de difficulté. La capacité du professionnel à s'affirmer face à ce qu'il pourrait considérer comme infondé ou injuste ne doit pas être sous-évaluée. Cette capacité peut s'exercer avec des familles mais elle est beaucoup plus délicate face à un comportement d'un résident très dépendant ou désorienté, qui sort de la norme. Nos résultats montrent que le comportement des familles n'est pas, même au niveau descriptif, une réelle source de stress. Certes, des événements surviennent au sein des structures et révèlent une insatisfaction des familles exprimée de manière virulente ou non, mais nous ne pouvons pas parler d'une altération du stress au sens de Seyle (1956). Les stratégies de coping centrées soit sur le problème, Lazarus et Folkman (1984) soit sur l'émotion, BruchonSchweitzer (2001), prennent alors tout leur sens. D'où un réel parallèle avec la notion de « locus of control » de Cooper et al. (1983).

\subsection{L'absence de latitude dans le processus de décision comme sources de perception du stress}

Nos résultats ont confirmé que l'individu est donc plus enclin à être stressé en raison de sa propre relation avec le groupe que par son positionnement face à des difficultés de groupe ou des clivages tels que nous l'avions identifié lors de la phase de pré test. Ce résultat fait écho à la théorie relative à l'organisation comme un construit social dynamique, de Crozier, Friedberg (1977) complétée par les travaux de Friedberg (1994). Cette relation influence également le degré d'investissement de la personne au sein de l'organisation, sa perception de l'environnement et la mise en avant de son pouvoir dit d'expertise.

D'un point de vue managérial, nous avons pu observer que le facteur « ordres contradictoires » ressort de la régression. Ainsi, les professionnels en EHPAD sont, non seulement confrontés à des ordres qu'ils estiment contradictoires, mais cette confrontation est source 
de stress perçu. Ces ordres contradictoires ont pu être analysés à travers le prisme de l'institution et notamment le travail réel propre à l'activité et le travail prescrit propre à la tâche. Cela rejoint les résultats de l'étude de Morin, Trepo, Grappy, Johnson (2013) sur la qualité de vie au travail au sein d'une clinique.

Enfin le dernier critère propre à l'encadrement est ressorti négativement de la régression, dans la mesure où ce sont les professionnels qui ne se considèrent pas comme encadrants qui ressentent plus fortement la notion de stress. Les explications à ce résultat sont multiples et peuvent s'expliquer à partir du modèle de Karasek (1979), qui lie la latitude de décision en fonction du degré d'autonomie de la personne au travail ainsi que de l'intensité du contrôle effectué sur son travail. Ainsi, un rythme très soutenu de l'activité, ou des interruptions fréquentes, sont autant de sources perturbatrices pouvant augmenter la demande psychologique. Dans nos résultats, le rythme et les interruptions dans le travail sont des facteurs qui figurent respectivement dans la régression aux première et sixième positions. Nous pouvons donc en déduire que les métiers qui sont en contact direct avec la personne accueillie demandent une exigence psychologique forte au regard des situations rencontrées (fin de vie, mort, aspects ingrats du métier...) alors que la latitude décisionnelle est faible. D'où cette conclusion d'une forte tension et d'un travail qui en effet peut être qualifié d'éreintant. Le modèle de Karasek et Theorell (1990) complète cette analyse par l'apport d'un nouveau déterminant qu'est le soutien social des collègues et des supérieurs en lien avec la mesure de l'intensité perçue de stress. Ainsi, au regard de nos résultats, l'encadrement n'apparaît pas comme facteur de stress. Au contraire, l'encadrant est plus enclin à disposer d'une latitude de décision lui permettant de ne pas se trouver dans une situation de « job strain ». Cette analyse renvoie alors aux corrélations qui existent entre les facteurs rythme, clarté, interruption et encadrement.

\section{DISCUSSION : QUELS LEVIERS DE GESTION DE LA PERCEPTION DU STRESS DANS LES STRUCTURES MÉDICO-SOCIALES ?}

Plusieurs leviers apparaissent comme opportunité de gestion du stress perçu par le professionnel, que nous synthétisons dans la figure ci-dessous :

Figure 3 : Facteurs d'influence de la perception du stress au sein de structures médico-sociales

\section{Tendre à faire coïncider le travail prescrit avec le travail réel (activité) en définissant une \\ stratégie concertée et en déduisant les priorités organisationnelles \\ Référence aux hypothèses $\mathrm{H} 3 \mathrm{~b}$ (ordres contradictoires), $\mathrm{H} 2 \mathrm{~b}$ (rythme) et H2e (interruptions)}

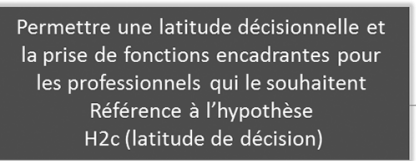

$\mathrm{H} 2 \mathrm{c}$ (latitude de décision)

Développer le sens du et au travail Référence à l'hypothèse

H3h (absence de sens au travail)
Perception du stress par le professionnel
Clarifier les missions de chaque professionnel mais aussi lui communiquer les missions de celles et ceux avec qui il travaille

Référence à l'hypothèse H3e (clarté de la mission)

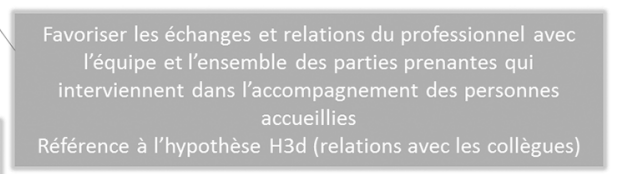

Veiller à l'influence du comportement des résidents sur le ressent

du professionnel et encadrer les situations à risque

Référence à l'hypothèse $\mathrm{H} 1 \mathrm{a}$ (comportement du résident) 


\subsection{Organiser l'activité et communiquer sur les missions individuelles et collectives}

La définition d'une stratégie adaptée à l'environnement consiste à faire coïncider le travail prescrit et réel. Cela signifie que l'activité devra continuellement être réévaluée, notamment avec des indicateurs tels que le GIR Moyen Pondéré (GMP) ou le Pathos Moyen Pondéré (PMP). Toutefois, les organisations doivent être capables de s'appuyer sur d'autres indicateurs visant à prendre en considération les troubles neuro psychiatriques, par exemple. Il est ainsi nécessaire de s'inscrire dans une logique d'évaluation globale de l'activité de l'établissement qui doit être effectuée à période régulière et plus fréquente pour adapter le rythme du professionnel (H2b), par exemple à un rythme trimestriel. Or, force est de constater que les évaluations de la dépendance demeurent rares, ou réalisées, soit dans le cadre des « coupes pathos » pour négocier la « dotation soin » d'un EHPAD, soit dans le cadre des reconductions annuelles budgétaires. Cette évaluation n'est pas suffisante pour garantir une adaptation de la stratégie à un environnement qui évolue rapidement. Les structures doivent se différencier et s'intégrer dans un contexte socio-économique de plus en plus complexe. Les Pôles d'Activités et de Soins Adaptés (PASA), mais aussi les Unités d'Hébergement Renforcés récemment instaurés en sont l'illustration. La combinaison avec un accueil de jour, permettant une ouverture sur son environnement est une bonne stratégie de différentiation par une combinaison des modes d'accueils proposés. D'où l'importance d'avoir une réflexion sur son insertion en amont via, par exemple, le lien avec des résidences autonomie dont les nouvelles missions sont clarifiées par décret ${ }^{1}$ et, en aval, via l'insertion dans un dispositif sanitaire intégré.
Il ressort un second levier propre à la clarté de la mission (H3e) de chacun des professionnels. Savoir s'il est bon ou non de stimuler une personne de plus en plus dépendante, ou au contraire de la protéger et faire en sorte qu'elle soit la moins sollicitée, est un facteur déterminant car ce sont ces situations qui peuvent générer une ambiguïté autour de la mission. Cette absence de clarté peut générer une perte de contrôle par l'encadrement (H3b). Il est donc fondamental pour un manager d'impulser une vision partagée de la mission autour de cette question.

Si elle n'est pas explicitée et affirmée clairement par l'encadrement, cette part de l'activité peut rapidement se transformer en partie cachée, au sens de Dejours (2000). Pour cela, le manager doit générer des principes de fonctionnement permettant de se centrer sur l'activité et éviter des interruptions qui perturbent le professionnel (H2e). Ces principes reposent sur le fait que la parole de chacun est d'égale valeur en reconnaissant qu'elle n'est pas d'égal effet. Cet ajustement mutuel est le moteur des structures et la compétence du manager réside dans sa capacité à savoir l'insuffler, l'entretenir et le dynamiser. D'où l'intérêt pour le manager des stratégies d'ajustement dans les tensions de rôle comme le soulignent Rivière, Commeiras \& Loubès (2013), dans leur étude au sein d'un CHRU.

\subsection{Générer des délibérations interdisciplinaires autour de l'accompagnement de la personne accueillie en intégrant la complexité des prises en charge globales}

Si les organisations appliquaient à la lettre tous les protocoles, ne laissant plus la place à

\footnotetext{
${ }^{1}$ Décret n ${ }^{\circ} 2016-696$ du 27 mai 2016 relatif aux résidences autonomie et portant diverses dispositions relatives aux établissements sociaux et médico-sociaux pour personnes âgées.
} 
l'ajustement mutuel ni à la marge d'adaptation, alors le processus de construction sociale lié à la régulation des organisations (Reynaud, 1997) en serait altéré. Des protocoles et un niveau de prescription raisonnable doivent être ainsi établis, mais ils doivent être ciblés, concis, réévalués et accessibles (Fraisse, Robelet, Vinot, 2003). Ces protocoles co-construits, et non imposés, pour favoriser les échanges entre professionnels (H3d) doivent avant tout définir un résultat attendu et non en premier lieu un processus. L'exemple le plus parlant porte sur la bientraitance. Il n'y a pas de protocole permettant de garantir la bientraitance. Or, cette absence permet justement une remise en question régulière de la structure. C'est la raison pour laquelle, nous proposons dans la continuité de Malo (2003) et Marion et al. (2012) un idéal-type de gestion globale par contrôle de la performance.

Cette analyse de la performance délivrée doit être au cœur des organisations, afin d'apporter des mesures correctives et de proposer plus aisément une ré évaluation du projet d'établissement ou une procédure d'évaluation et des suggestions visant à améliorer la prestation. Ceci illustre une démarche d'évaluation de la prestation délivrée avec la personne directement concernée, par le projet d'accompagnement individualisé qui suppose un contrôle, non du processus, mais du résultat. Ainsi, l'organisation serait plus à même de se centrer sur la personne accueillie, qui est in fine le cour de sa mission. Elle permettrait au professionnel d'exprimer de manière constructive et partagée les difficultés ressenties qui pourraient altérer sa mission et les facteurs constitutifs d'un stress (H1a).

\subsection{Favoriser la prise d'initiative et générer du sens}

La latitude de décision (H2c) et le sens au travail (H3h) sont des leviers sur lesquels le manager peut s'appuyer pour influencer la perception du stress. C'est en favorisant le sentiment d'être en capacité à pouvoir décider et d'être acteur que le professionnel s'estimera plus à même de lutter face à de potentiels facteurs de stress. Cela peut assurément passer par une gestion du mode de l'erreur. Tout l'art du manager va alors consister à gérer cette erreur pour renforcer la vision commune et valoriser l'ajustement mutuel. Dans le secteur sanitaire et médico-social, les professionnels accordent une importance toute particulière à la notion d'engagement, de service et pour qui, le sens de leur action doit pouvoir se concilier avec des valeurs personnelles. Une attention particulière doit alors être apportée aux signes de reconnaissance positifs, qu'ils soient ou non verbaux. Il est important de valoriser les conséquences de l'engagement du professionnel sur la qualité de l'accompagnement offert à la personne accueillie, afin de développer la prise d'initiative et le sentiment pour le professionnel d'avoir une emprise sur des facteurs qui peuvent être ressentis comme vecteurs de stress.

\section{CONCLUSION}

Cette recherche a permis d'identifier les facteurs pouvant constituer des leviers d'action sur la réduction du stress chez le professionnel. Nous avons pu dégager 8 facteurs de la régression linéaire multiple de la fonction perception du stress en EHPAD, qui sont respectivement par ordre d'importance : le rythme de travail, la manque de clarté dans les missions de chacun, les interruptions fréquentes dans le travail, le sens au travail, le comportement des résidents, les relations avec ses collègues, les ordres contradictoires, le fait de ne pas occuper une fonction d'encadrement.

D'un point de vue méthodologique, cette recherche a tout d'abord mis en avant 
la précaution qui doit être déployée lorsque la notion de stress au travail est abordée. L'approche plurielle en est une des raisons tout comme les spécificités propres à chaque individu, son âge, son métier, sa formation, sa santé, son soutien social, sa culture, ses croyances, sa situation économique...

Cette recherche revêt toutefois plusieurs limites. En premier lieu, le stress, qu'il soit autoévalué ou mesuré scientifiquement par PSS 10, reste d'abord porté par une évaluation subjective de l'individu. D'autres méthodologies pourraient relever des éléments non verbaux ou non-dits mais objectivement mesurables et observables. Les enquêtes de terrain comme s'en inspire par exemple l'ethnologue Althabe (1990) pourraient constituer une piste en valorisant de facteurs jusqu'ici non exprimés. La question du contre-transfert du chercheur dans une position, in fine d'ethnologue, aurait pu être traitée tout comme la personnalité sociale du chercheur, Girin (1989).

Par ailleurs, cette recherche nous a conduit à proposer un modèle en cinq points qui s'appuie sur la définition d'une stratégie adaptée à l'environnement au regard de son état actuel et futur, une organisation professionnelle qui se superpose à sa stratégie, le développement de la performance sur la prestation délivrée, le développement de la performance sociale et le développement de la performance économique. Un prolongement utile tendrait à pouvoir tester les hypothèses ouvertes par ce modèle de performance. Ainsi, et plus spécifiquement, la question de la perception du stress au regard d'une performance tournée vers la prestation délivrée, vers la performance sociale et vers la performance économique serait posée dans un contexte de plus en plus contraint.

\section{RÉFÉRENCES BIBLIOGRAPHIQUES}

Alis D., Conflits de rôles et régulations autonomes du personnel en contact avec la clientèle : le cas des agents généraux d'assurance, université de Aix-Marseille 3, 1997.

Althabe G., Ethnologie du contemporain et enquête de terrain, Terrain, 1990 : 126-131.

Aubry F., Conséquences sur la santé au travail de rythmes temporels contradictoires, en France et au Québec, Revue de Sciences Sociales et Humaines, 2012.

Bellinghausen L., Collange J., Botella M., Emery J-L., Albert E., «Validation factorielle de l'échelle française de stress perçu en milieu professionnel », Santé Publique 4/2009 (Vol. 21) : 365-373.

Carrier Vernhet A., Desmarais C., L'implication organisationnelle peut-elle accentuer les effets du stress sur le mal-être ? AGRH Septembre 2012.

Codo S, Le stress professionnel des managers intermédiaires et des managers de proximité : une application au secteur public, thèse de doctorat en sciences de gestion, Université François RABELAIS de Tour, 2012.

Cohen S., Kamarck T., Mermelstein R., Global measure of perceived stress. Journal of Health and Social Behavior, 1983 : 385-396.

Cooper, Harris M., Findley, Maureen J., Locus of control and academic achievement: A literature review, Journal of Personality and Social Psychology, Vol 44(2), Feb 1983 : 419-427.

Crozier M., Friedberg E., L'acteur et le système, Paris, Seuil, 1977.

Crozier M.,L'Entreprise à l'écoute, Seuil Points Essais, numéro 279, 1994.

Dejours C., Souffrance au travail, nouvelles pathologies et nouvelles réponses. Le concours médical, Entretien à partir des propos recueillis par Martine Lochouarn, 2000 : 518-521.

Demerouti E., Geurts SA, Bakker AB, Euwema M., The impact of shiftwork on work--home conflict, job attitudes and health, Ergonomics, 2004 : 987-1002.

Dupuis G., Martel J.P., Voirol, C., et al. La qualité de vie au travail : Bilan de connaissances, 2009 
Geurts S.A.E., Kompier M.A.J., Roxburh S., Houtman I.L.D., Does work home interference mediate the relationship between workload and well being? Journal of vocational behavior, 2003 : 532-559.

Fraisse, S., Robelet, M., Vinot, D., La qualité à l'hôpital : entre incantations managériales et traductions professionnelles, Revue Française de Gestion, $\mathrm{n}^{\circ} 146,155-166,2003$.

Gadrey, J., Nouvelle économie, nouveau mythe, Paris, Flammarion, 2000.

Gintrac A., Le stress au travail, un état des lieux, Revue Management et avenir, $\mathrm{N}^{\circ} 41$, 2011:89-106.

Girin J., Quel paradigme pour la recherche en gestion ?, Economie et santé, série sciences de gestion $\mathrm{N}^{\circ} 2,1981: 1872-1889$.

Guillet L, Le stress, De Boeck, 2012.

Karasek R., Job demands, job decision latitude, and mental strain: Implications for job redesign. Administrative Science Quaterly, 1979 : 285-308.

Hellemans C., Karnas G., Epreuve de validation du modèle de Karasek auprès de travailleurs du secteur tertiaire. Relations du modèle avec les tensions mentales. Revue Européenne de Psychologie appliquée, volume 49, n³, 1999 : 215-224.

Junker, B., The field work situation, Social roles for observation, University of Chicago Press, 1960.

Karasek R., Theorell T., Stress, productivity, and the reconstruction of working life, New york, Basic Book, 1990.

Kahn R.L., Role conflict and ambiguity in organizations, The Personnel Administrator, 9, 1964.

Katz R., Kahn R.L., The Social Psychology of Organizations, Wiley \& son, 1966.

Kolek M., Bruchon-Schweitzer M., Bourgeois M-L., stress et coping : un modèle intégratif en psychologie de la santé, Annales médico psychologiques, 2003 : 809-815.

Lazarus R, Folkman S, Stress, appraisal and coping, Springer Publishing Company, New York, 1984.

Legault M-J, Belarbi-Basbous, H., Gestion par projets et risques pour la santé psychologique au travail dans la nouvelle économie. Université du Québec à Montréal, 2006.
Loubès A., « Contribution à l'étude des tensions de rôle des agents de maîtrise en milieu industriel - Une étude empirique », Thèse de Doctorat en Sciences de Gestion, Université de Montpellier II, soutenue le 7 janvier 1997.

Marion, A., Asquin, A., Everaere, C., Vinot, D., Wissler, M., Diagnostic de la performance de l'entreprise, Concepts et methods, Dunod, Paris, 2012.

Minvielle E., Aegerter P., Dervaux B., Boumendil, A., Retbi A., Jars M-C., Guidet., Assessing organizational performance in intensive care units : A french experience, Journal of critical Care, $\mathrm{n}^{\circ} 23,2008$ : 236-244.

Moisson M., Fuhrer C., Cucchi A., Les déterminants du stress et de l'épuisement professionnel, $\mathrm{XXI}^{\mathrm{e}}$ congrès de l'AGRH, Rennes-Saint Malo, 2010.

Morin E., Trepo G., Grappy A-C, Johnson K., Enquête sur la qualité de vie au travail à l'institut Robert Merle d'Aubigne, HEC Montréal, 2013.

Morin E., Gagne C., Donner un sens au travail et promouvoir le bien-être psychologique, Guide pour les gestionnaires et les intervenants, 2009.

Pauget B., L'arrivée de la génération Y : quelles conséquences managériales et organisationnelles pour les organisations sanitaires et sociales françaises ?, Pratiques et Organisation des Soins 1/2012 (Vol. 43) : 25-33.

Perrot S., Nature et conséquences des conflits de rôles, Journée de recherche CEROG / AGRH, IAE d'Aix, Aix en Provence, Actes en ligne, 2005.

Ponnelle S., Lancry A., Stratégies d'ajustement et ressources environnementales et personnelles dans la dynamique du stress, in M. Neboit, M. Vézina, Stress au travail et santé psychique, Toulouse, Octarès, 2002.

Pronost AM., Legouge A. Leboul D., Gardembas-Pain M., Berthou C., Giraudeau B., Colombat P.,Effet des caractéristiques des services en oncohématologie développant la démarche palliative et des caractéristiques sociodémographiques des soignants sur les indicateurs de santé: soutien social, stress perçu, stratégies de coping, qualité de vie au travail, Oncologie, Volume 10, Issue 2, 2008: 125-134.

Reynaud, J.-D., Les règles du jeu - l'action collective et la régulation sociale, A. Colin, Paris, 1997 (rééd.). 
Rivière A., Commeiras, N., Loubès, A., Tensions de rôle et stratégies d'ajustement : une étude auprès de cadres de santé à l'hôpital, Journal de Gestion et d'Economie Médicales, 2013, volume 31.

Selye, H., A syndrome produced by diverse Nocuous Agents, Nature, 1936 : 32-38.

Seyle H., The stress of life, McGraw-Hill, New York, 1956.

Siegrist J., Adverse health effects of high effort / low reward, Journal of Occupational Health Psychology, Vol 1(1), Jan 1996 : 27-41.

Steiler D., Rosnet E., La mesure du stress professionnel : Différentes méthodologies de recueil. Revue des Sciences de Gestion, 2011: 271-279.
Timstit-Berthier M., Evolution du concept de stress : de la physiologie à la victimologie, Res-Systemica, Vol. 10, Mai 2014.

Vinot A., Le stress du professionnel en EHPAD au regard de l'influence de la satisfaction des résidents et des familles : étude quantitative et qualitative au sein de 26 EHPAD, Thèse de doctorat en sciences de gestion, Université de Lorraine, 18/11/2013.

Voydanoff P., Work demands and work-to-family and family-to-work conflict direct and indirect relationships, Journal of family issues, 26 (6) : 707-726. 